\title{
Development of a Technique for Energy Storage Using a Piezoelectric Generator for Low-Power Consumption Devices Supporting Stand-Alone Wireless Sensors for Smart Systems
}

\author{
Panu Thainiramit ${ }^{1, *}$, Yufridin Wahab ${ }^{2}$, Masri Zairi Mohd Zin ${ }^{2}$, Eleeyah Saniso ${ }^{3}$, \\ Kua-anan Techato ${ }^{1}$, Nantakan Muensit ${ }^{4,5}$
}

${ }^{1}$ Faculty of Environmental Management,

Prince of Songkla University (PSU), Hat Yai, Songkhla, 90112, THAILAND

${ }^{2}$ Advanced Multidisciplinary MEMS-Based Integrated Electronic NCER Centre of Excellent (AMBIENCE), School of Microelectronic Engineering,

Universiti Malaysia Perlis, Kuala Perlis, Perlis, 02000, MALAYSIA

${ }^{3}$ Program of Renewable Energy Technology, Faculty of Sciences Technology and Agriculture,

Yala Rajabhat University, Yala, 95000, THAILAND

${ }^{4}$ Department of Physics, Faculty of Science,

PSU, Hat Yai, Songkhla, 90112, THAILAND

${ }^{5}$ Center of Excellence in Nanotechnology for Energy (CENE),

PSU, Hat Yai, Songkhla, 90112, THAILAND

*Corresponding Author

DOI: https://doi.org/10.30880/ijie.2019.11.01.006

Received 27 May 2018; Accepted 20 August 2018; Available online 30 April 2019

\begin{abstract}
In the last decade, microcontrollers (MCU) have become part of daily life. MCU can function as standalone devices to control automatic machines in so-called Smart Embedded Systems (SEMs) which can operate independently using Direct Current (DC) power. Such systems actually need a conventional battery to function and battery lifetime is a major problem since it is not easy to monitor when exhausting the power. This research can solve the problem, how to keep the SEM continually functioning by using piezoelectric power generator to charge the rechargeable battery through the enhancement circuit technique. This is described by the piezo-current and piezo-voltage transferred into a small rechargeable battery. The piezo-power is generated under the constant vibration based on ambient-source movement. The rechargeable battery, capacity $150 \mathrm{mAh}$, can be fully charged within 40 hours. Moreover, this paper presents the theoretical and experiment results from the output power of the piezo-generator. The results confirm that DC-DC converter technique can validate the model representation and achieve the power enhancement of piezoelectric generator.
\end{abstract}

Keywords: piezoelectric material, power condition, buck-boost converter, DC-DC power conversion, rechargeable battery, energy conversion, energy harvesting.

\section{Introduction}


The idea that piezoelectric materials to be able to convert mechanical energy into electrical energy by external mechanical force is not recently new but, in practice, piezoelectric generators produce extremely low power with the energy range usually around a milliwatt or below (Shenck \& Paradiso, 2001; Thainiramit, 2013). The main consideration in using piezoelectric materials is their mechanical properties and their ability to produce electric power. Ceramic materials, for instance, accept large stresses but their strains are very small. However, piezo-ceramics are able to operate at low mechanical frequencies and it is possible to use these materials effectively at ambient mechanical vibrations in the range of 1 to $100 \mathrm{~Hz}$ (Roundy, Wright, \& Rabaey, 2003).

Recent developments in wearable electronics and wireless systems require more energy and use conventional batteries, rechargeable batteries and super-capacitors to store electrical energy and to maintain a reserve power capacity in case of an emergency. An electrical technique using a DC-DC converter was presented by Ottman et al. (Ottman, Hofmann, Bhatt, \& Lesieutre, 2002; Ottman, Hofmann, \& Lesieutre, 2002), using a buck converter to charge a rechargeable battery. Moreover, this technique used a switching regulator principle which can match the impedance of the load resistance within the rechargeable battery (Hart, 2011).

This paper is focused on power optimization from vibration and the proposed technique. These are implemented by employing the direct force excitation. Power optimization is achieved by an electromechanical device using as a DC generator (Guyomar, Badel, Lefeuvre, \& Richard, 2005). The circuit interface employs a buck-boost DC-DC converter and the power is generated from this circuit interface using its switching mechanism. The power output from a theoretical prediction based on the charging state of a specific rechargeable battery was validated by experimental results. The paper presents graphs illustrating the characteristics of the charging state to confirm that the battery can be fully charged using the piezoelectric generator.

\section{Piezoelectric Generator}

According to the IEEE standards relating to piezoelectricity, the piezoelectric equations can be written as:

$$
\left\{\begin{array}{l}
T=c S-e E \\
D=e S+\varepsilon E
\end{array}\right.
$$

where $T$ is the stress, and $S$ is the strain. $E$ is the electric field, and $D$ is the electrical induction. $\varepsilon$ is the clamped permittivity, and $e$ is the piezoelectric coefficient.

The piezoelectric generator equations $(2)$ can be linked to the mechanical variables $\left(y, F_{P}\right)$. The electrical variables $(I, V)$ can thus be rewritten into the consideration motion as (Roundy, Wright, \& Pister, 2002):

$$
\left\{\begin{array}{l}
F_{P}=K_{E} y+\alpha V \\
I=\alpha \dot{y}-C_{P} \dot{V}
\end{array}\right.
$$

Where $F_{P}$ is the piezoelectric restoring force, and $I$ is the piezoelectric current. These two variables are under $y$, which is the vibration direction. $C_{P}$ is the material capacitance related to the $\alpha$, which is the applied force, and $K_{E}$ is the stiffness of the piezo-element.

Electrical power $P$ is produced by the piezo-generator through sinusoidal excitation, which is a steady-state operation. The displacement of the structure at the operation frequency $\omega_{0}$ is the constant magnitude $F_{M \text {,Yconst }}$. The power output equation is given below in (3). This equation shows that the power output from piezo-generator is related to the load resistance and the figure of merit $(\mathrm{FoM})$, where FoM $=\left(k_{t}\right)^{2} Q_{s t r}$. The electromechanical coupling factor $\left(k_{t}\right)^{2}$ corresponds to the material's conversion property and the structural quality factor $Q_{s t r}$ is the harvested structure property corresponding to the structural vibration capturing the available mechanical energy.

$$
P=\begin{array}{cc}
R \alpha^{2} & F_{M, \text { Yconst }}^{2} \\
\left(C+\left(2 R \alpha^{2} /\left(R C_{P} \omega_{0}+(\pi / 2)\right)^{2}\right)\right)^{2}\left((\pi / 2)+R C_{P} \omega\right)^{2}
\end{array}
$$

The output power thus resulting is presented in Fig. 1 which summarizes the normalized power variation as a function of the normalized load resistance and as a function of varied FoM parameters. Infrequently operation, the generated power is proportional to the magnitude of the piezo-generator depended on the value of the load resistance $R$. The load resistance maximizes the power at two peaks of the optimum resistance value depended on the FoM. 


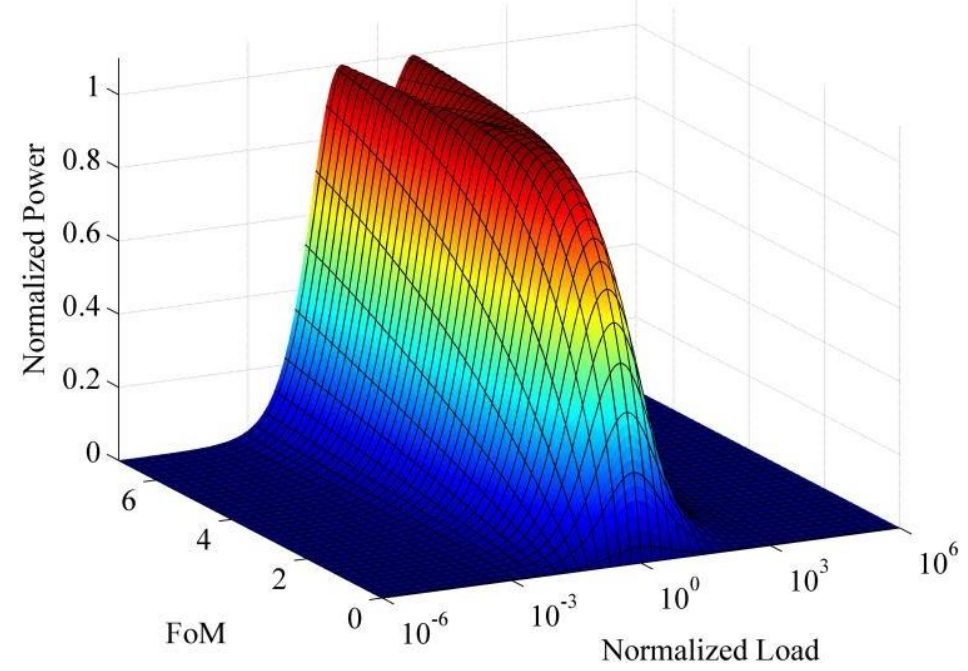

Fig 1. - Normalized power as a function of the normalized resistance and the FoM at the operation frequency of the harvested structure.

\section{Power Optimization Technique}

Based on the model presented in the previous section, the power of a piezo-generator is strongly dependent on the load resistance. The power is at a maximum for one or two matching optimum loads. The FoM needs to be greater than 2 in order to charge a rechargeable battery (Sodano, Park, Leo, \& Inman, 2005). The values are determined by the piezo-generator based on its electromechanical characteristics and depending on the particular vibration frequency of the ambient excitation source (Guyomar et al., 2005; Rakbamrung et al., 2010).

The piezo-generator is a simple cantilever beam formed from the piezoelectric material. The beam structure can capture ambient vibration sources based on their resonance frequencies, and the operating frequency is defined at around the near resonance. Therefore, the beam structure is able to produce the maximum power output and a DC output can be obtained using a rectifier circuit, as shown in Fig. 2.

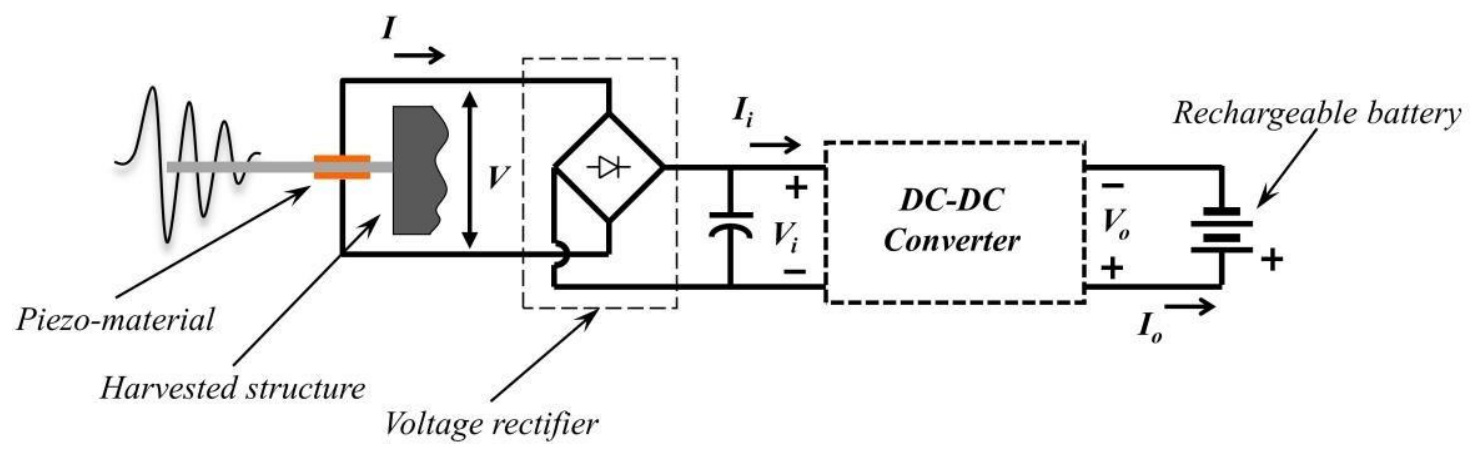

Fig. 2. - Schematic of this circuit interface for charging the rechargeable battery using the piezoelectric material as a generator.

Generally, the maximum electrical power from the single piezoelectric generator is unable to charge a rechargeable battery, even when the piezo-voltage is higher than the battery voltage (Hart, 2011; Thainiramit, 2013). To solve this problem, a buck-boost circuit is applied. This paper presents the output power $P$ of an experimental investigation employing the Continuous Current Mode (CCM), and the relevant parameters of the Discontinuous Current Mode (DCM) can be derived from the CCM. The CCM can be implemented in the piezo-electric parameters based on the electrical threshold condition with both minimum current and inductor size as well as load resistance related to the output power.

\subsection{Harvesting Circuit Technique}

The schematic of the buck-boost circuit is presented in Fig. 3 as only simple components following the circuit principle. This figure is shown that operation states of its current transfer are very crucial property, which is represented by the inductor current $I_{L}$. This technique is able to be isolated completely between input and output within different both sides. 


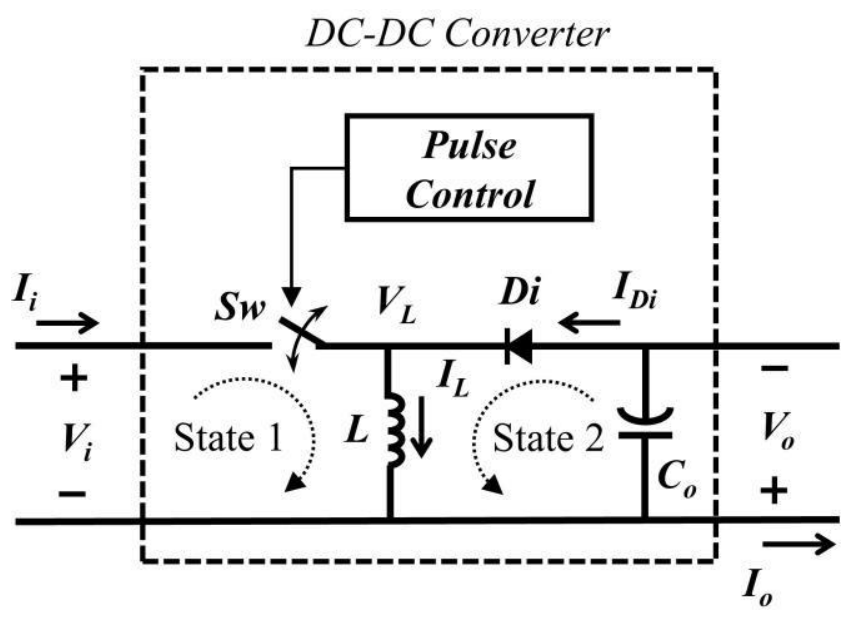

Fig. 3. - Buck-boost converter circuit.

The State 1 of the CCM corresponding to the charging process of the inductor $L$ will be activated by pulse control unit connected to the switch $S w$ by conditions of the duty-ratio $\delta$ ( $\delta$ denotes that the circuit is closed, the switch turns on $T_{o n}$. $(1-\delta)$ denotes that the circuit is opened, the switch turns off $\left.T_{o f f}\right)$. The inductor voltage $V_{L}$ is defined as a $V_{i}$, which is the piezoelectric voltage $V$. The piezoelectric current $I$ can be represented at the same time by the input current $I_{i}$ and inductor current $I_{L}$. This current is flowing through the switch $S w$ during the status $T_{o n}$.

After the switch turns off $T_{o f f}$, the load resistance will be applied. In this state, the inductor voltage $V_{L}$ is becoming the $V_{o}$. This output voltage $V_{o}$ is to be the piezoelectric voltage $V$, which is applied to the load resistance or rechargeable battery in this system. Afterwards, the State 2 of the CCM is completely employed in another side of the converter. The output voltage $V_{o}$ is still drawn to the load from the inductor energy. The diode $D i$ is forward biased due to the current direction of the State 2. The smooth capacitor $C_{o}$ should be designed for its output current $I_{o}$ as a low ripple signal. The diode $D i$ should be also designed for the high-frequency switching devices as well. Hence, the expression of the output voltage $V_{o}$ as a function of the input voltage $V_{i}$ and the duty-ratio $\delta$ is given by

$$
\left.V=V{ }_{\text {in }} \mid \begin{array}{c}
\delta \\
\delta-1
\end{array}\right) .
$$

Most applications in which a buck-boost converter may be used within the high power consumption. The output current $I_{o}$ of this system related to the diode current $I_{D i}$ and the inductor current $I_{L}$ is given by

$$
I_{o}=I_{L}-I_{D i} \text {. }
$$

If the diode current $I_{D i}$ reducing to the minimum value is more than or equal zero, the optimum value of the inductor, $L_{o p t, C C M}$, for the CCM can be written by

$$
L_{o p t, C C M}>\frac{R(1-\delta)^{2}}{2 f_{s w}} .
$$

Hence, in the case of the DCM, the inductor current may be lower than zero. The optimum value of the inductor, $L_{o p t, D C M}$, for the DCM can be given by

$$
L_{o p t, D C M}<\frac{R(1-\delta)^{2}}{2 f_{s w}} .
$$

In this application, to charge the rechargeable battery can be verified by these designed parameters to implement into the circuit technique. Therefore, the variation of the voltage output $V_{o}$ as a function of the duty-ratio $\delta$ is able to be defined by this expression, 


$$
\frac{d V_{o}}{d \delta}=-V \sqrt[i]{\frac{R}{2 f_{s w} L}} .
$$

\section{Experimental Result}

This section describes the result of the experiment in terms of the electrical power produced from the buck-boost circuit. This circuit is able to charge a rechargeable battery with an appropriate FoM value. The results confirm that the output is coming from the enhancement technique, which is able to be applied in the high-performance power range and the FoM value greater than 2 was found when using the switching technique, as shown in Fig. 4.

(a)

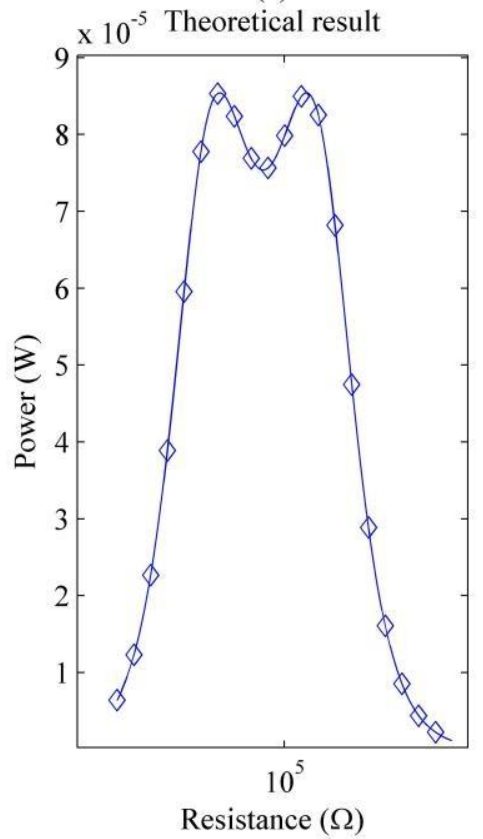

(b)

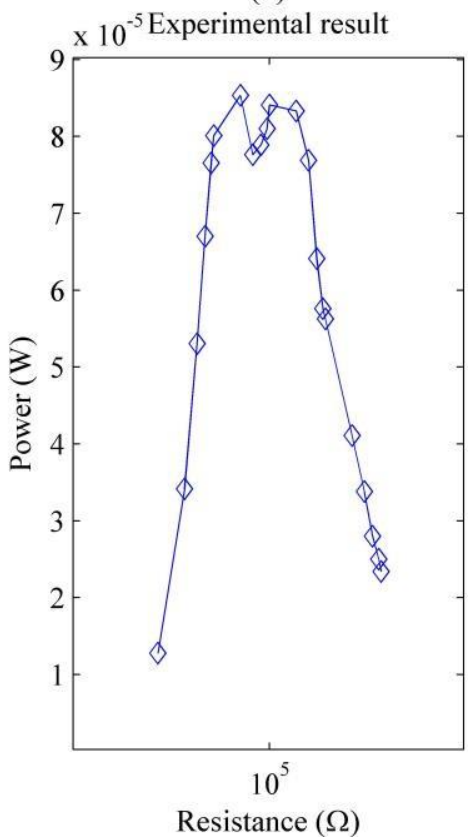

Fig. 4. - Experimental and theoretical result from harvested power using the DC-DC converter circuit.

Fig. 5 shows the results in terms of the voltage and current supplied to the rechargeable battery when it is fully charged. This result was obtained under CCM conditions and also followed the minimum-starting-state rule for the charge accumulation into the rechargeable battery.

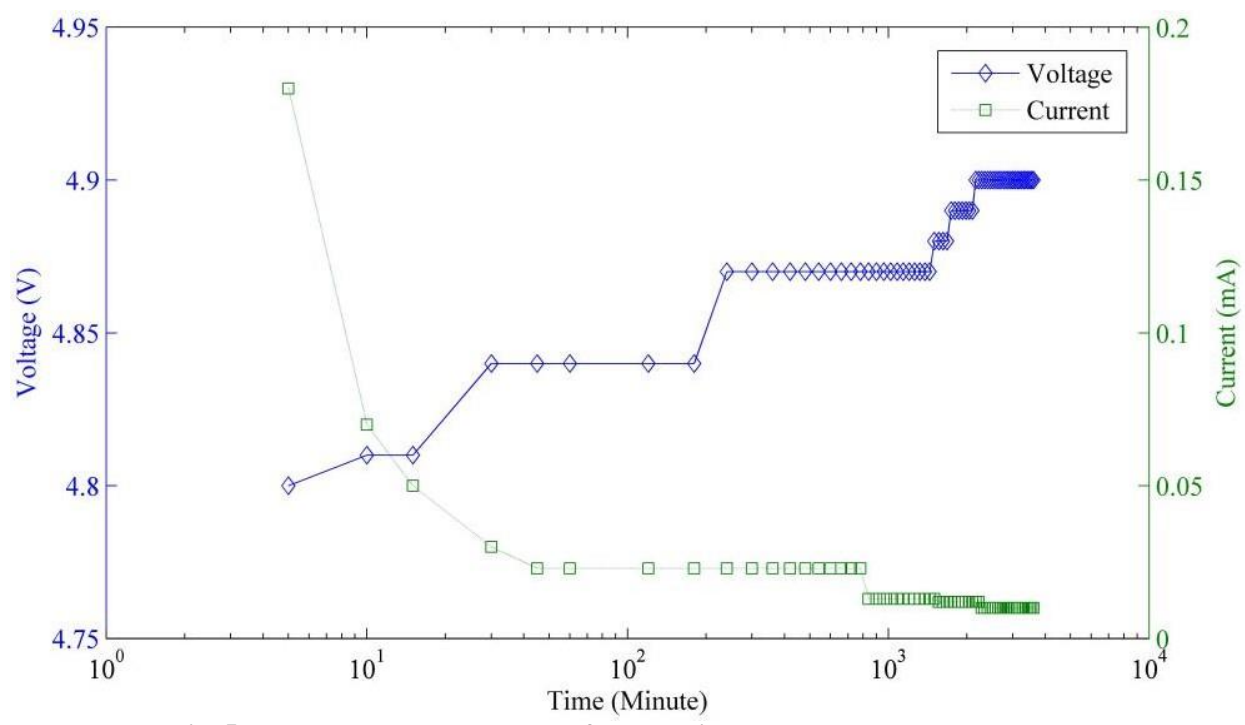

Fig. 5. - Voltage and current when full charging the rechargeable battery. 


\section{Conclusion}

This paper focuses on the optimization of the power from a directly applied force on a piezoelectric device. The energy harvester converts ambient vibrations into electrical energy and the piezo-generator may be used as the specific power source for a rechargeable battery. The power optimization was based on the equations relating to piezoelectric materials, specifically the piezo-generator equation, and these electrical circuits composed of a full-wave rectifier, a capacitor for smoothing the voltage, the DC-DC converter technique and various resistors. In this study, the switching technique was used a buck-boost converter, which has the appropriate properties to optimize the power based on the relationship between the input voltage and the input current. This device was able to fully charge a $150 \mathrm{mAh}$, rechargeable battery in 40 hours due to the properties of the piezoelectric material. This piezo-generator can act as the electrical power source for electronic devices using a low power system and the characteristic charging graph confirmed the charging capacity of the buck-boost circuit. However, this technique is not suitable for use with a low applied force or a low electromechanical coupling factor and the circuit's intrinsic properties must be defined for power optimization. Furthermore, the power consumption of the buck-boost circuit can easily be minimized and the circuit requires neither sensors nor a sophisticated algorithm.

The developed system may be helpful in the design of the Integrated Circuit (IC) because the circuit interfaces are capable of optimizing power for miniature generators. Energy storage devices can use this technique to obtain the electrical charge from very-low-power generators. The developed circuit is also suitable for the power consumption of very-small devices using electrical power from other generator sources and future studies. The studies should be investigated the use and the further optimization of electromagnetic, thermoelectric, and photovoltaic energy harvesters.

\section{Acknowledgement}

This work has financially supported by The Center of Excellence in Nanotechnology for Energy (CENE), Prince of Songkla University who also provided the laboratory facilities.

\section{References}

[1] Shenck, N. S., \& Paradiso, J. A. (2001). Energy scavenging with shoe-mounted piezoelectrics. IEEE Micro, 21(3), 30-42.

[2] Thainiramit, P. (2013). Developments of Energy Harvesting Using the Piezoelectric Methods. (Master's thesis). Prince of Songkla University, Songkhla, Thailand.

[3] Roundy, S., Wright, P. K., \& Rabaey, J. (2003). A study of low level vibrations as a power source for wireless sensor nodes. Computer Communications, 26(11), 1131-1144.

[4] Ottman, G. K., Hofmann, H. F., Bhatt, A. C., \& Lesieutre, G. A. (2002). Adaptive piezoelectric energy harvesting circuit for wireless remote power supply. IEEE Transactions on Power Electronics, 17(5), 669-676.

[5] Ottman, G. K., Hofmann, H. F., \& Lesieutre, G. A. (2003). Optimized piezoelectric energy harvesting circuit using step-down converter in discontinuous conduction mode. IEEE Transactions on Power Electronics, 18(2), 696-703.

[6] Hart, D. W. (2011). Power Electronics: McGraw-Hill.

[7] Guyomar, D., Badel, A., Lefeuvre, E., \& Richard, C. (2005). Toward energy harvesting using active materials and conversion improvement by nonlinear processing. IEEE Transactions on Ultrasonics, Ferroelectrics, and Frequency Control, 52(4), 584-595.

[8] Roundy, S., Wright, P. K., \& Pister, K. S. J. (2002). Micro-Electrostatic Vibration-to-Electricity Converters. ASME 2002 International Mechanical Engineering Congress and Exposition Microelectromechanical Systems, November 17-22, New Orleans, Louisiana, USA, (36428), 487-496.

[9] Sodano, H. A., Inman, D. J., \& Park, G. (2005). Comparison of Piezoelectric Energy Harvesting Devices for Recharging Batteries. Journal of Intelligent Material Systems and Structures, 16(10), 799-807.

[10] Rakbamrung, P., Lallart, M., Guyomar, D., Muensit, N., Thanachayanont, C., Lucat, C., \& Sukwisut, P. (2010). Performance comparison of PZT and PMN-PT piezoceramics for vibration energy harvesting using standard or nonlinear approach. Sensors and Actuators A: Physical, 163(2), 493-500. 